\title{
Band bending at the surfaces of In-rich InGaN alloys
}

\author{
L. R. Bailey, ${ }^{1}$ T. D. Veal, ${ }^{1}$ P. D. C. King, ${ }^{1}$ C. F. McConville, ${ }^{1, a)}$ J. Pereiro, ${ }^{2}$ J. Grandal, ${ }^{2}$ \\ M. A. Sánchez-García, ${ }^{2}$ E. Muñoz, ${ }^{2}$ and E. Calleja ${ }^{2}$ \\ ${ }^{1}$ Department of Physics, University of Warwick, Coventry CV4 7AL, United Kingdom \\ ${ }^{2}$ ISOM and Departamento de Ingeniería Electrónica, ETSI Telecomunicación, Universidad Politécnica \\ de Madrid, Ciudad Universitaria, 28040 Madrid, Spain
}

(Received 16 July 2008; accepted 14 October 2008; published online 8 December 2008)

\begin{abstract}
The band bending and carrier concentration profiles as a function of depth below the surface for oxidized $\operatorname{In}_{x} \mathrm{Ga}_{1-x} \mathrm{~N}$ alloys with a composition range of $0.39 \leq x \leq 1.00$ are investigated using $\mathrm{x}$-ray photoelectron, infrared reflection, and optical absorption spectroscopies, and solutions of Poisson's equation within a modified Thomas-Fermi approximation. All of these InGaN samples exhibit downward band bending ranging from 0.19 to $0.66 \mathrm{eV}$ and a high surface sheet charge density ranging from $5.0 \times 10^{12}$ to $1.5 \times 10^{13} \mathrm{~cm}^{-2}$. The downward band bending is more pronounced in the most In-rich InGaN samples, resulting in larger near-surface electron concentrations. (C) 2008 American Institute of Physics. [DOI: 10.1063/1.3033373]
\end{abstract}

\section{INTRODUCTION}

Group III-N semiconductors have many fundamental properties that make them promising for use in optoelectronic and electronic devices. The ternary InGaN alloys have intrinsic band gaps that span a wide range of energies, from 0.64 (Ref. 1) to $3.44 \mathrm{eV}^{2}$, enabling a device to be produced by a single material system that covers a large optical window. ${ }^{3,4}$ It has also been reported that $\mathrm{GaN}$ has a high efficiency of luminescence ${ }^{5}$ and that $\mathrm{InN}$ has a small electron effective mass and high electron drift velocity, ${ }^{6,7}$ giving this semiconductor system excellent optical and transport properties. To produce high-speed electronic or optoelectronic devices from InGaN, more needs to be understood about the interface properties, such as how to form reliable contacts to the surface and how to control the surface sheet charge density. Therefore several investigations into the surface electronic properties of InGaN have been carried out in the past few years. ${ }^{4,8-12}$

Usually an electron depletion layer is observed at the surface of $n$-type III-V semiconductors. However, $n$-type InAs and InN exhibit a surface electron accumulation layer. ${ }^{11,13,14}$ This can be explained by the extremely low conduction band minimum $(\mathrm{CBM})$ at the $\Gamma$ point in both $\mathrm{InN}$ (Refs. 10 and 15) and InAs. ${ }^{14,16}$ Hence the branch point energy, defined as the crossover point from states that are predominantly acceptorlike to states that are predominantly donorlike, is located high in the conduction band of $\mathrm{InN}$. Surface states will be primarily donorlike (acceptorlike) when the Fermi level is below (above) the branch point energy. For InN, the Fermi level position is usually below the branch point energy, enabling the existence of unoccupied donorlike surface states. These positively charged states pin the surface Fermi level position near the branch point energy, resulting in the Fermi level increasing at the surface of $\mathrm{InN}$. Alternatively, this can be viewed as the conduction and va-

${ }^{a)}$ Electronic mail: c.f.mcconville@warwick.ac.uk. lence bands bending downward with respect to the Fermi level at the surface of InN. Hence an electron accumulation layer is formed at the surface of InN.

Previously, x-ray photoelectron spectroscopy (XPS) has been performed on $n$-type InGaN samples to determine the surface Fermi level position as a function of composition. ${ }^{8,12}$ Veal et al. ${ }^{8}$ reported that the surface Fermi level position varies from high above the CBM in InN to significantly below the CBM in GaN with the surface Fermi level position and branch point energy of $\operatorname{In}_{x} \mathrm{Ga}_{1-x} \mathrm{~N}$ intersecting at a composition of approximately $x=0.43 ., 12$ Without knowledge of the bulk Fermi level position, this intersection point was taken to be the composition at which a transition from surface electron accumulation to depletion occurs. This is a reasonable first approximation, but the extent of downward band bending can only be obtained from knowledge of both the surface and bulk Fermi level positions.

This paper reports a detailed investigation of the surface and bulk Fermi level positions for a range of compositions of InGaN using XPS and infrared reflection and optical absorption spectroscopies. Detailed knowledge of both the surface and bulk Fermi level positions enables quantitative determination of the behavior of the surface space charge region as a function of composition. To examine the band bending and carrier concentration profile as a function of depth below the surface, Poisson's equation was solved within a modified Thomas-Fermi approximation (MTFA).

\section{EXPERIMENTAL DETAILS}

Samples of $200 \mathrm{~nm}$ thick $\operatorname{In}_{x} \mathrm{Ga}_{1-x} \mathrm{~N}$ were investigated with a composition range of $0.39 \leq x \leq 1.00$. The InGaN samples were grown by plasma assisted molecular beam epitaxy (MBE) on an $80 \mathrm{~nm}$ thick GaN buffer layer, grown by MBE, on a template that consists of a $3 \mu \mathrm{m}$ thick Si-doped GaN layer, grown by metal-organic vapor phase epitaxy, and on a $330 \mu \mathrm{m}$ thick (0001) sapphire substrate. In the previous investigation of InGaN space charge region behavior, Veal et $a l .{ }^{8}$ used samples with a large range of thicknesses, from 100 


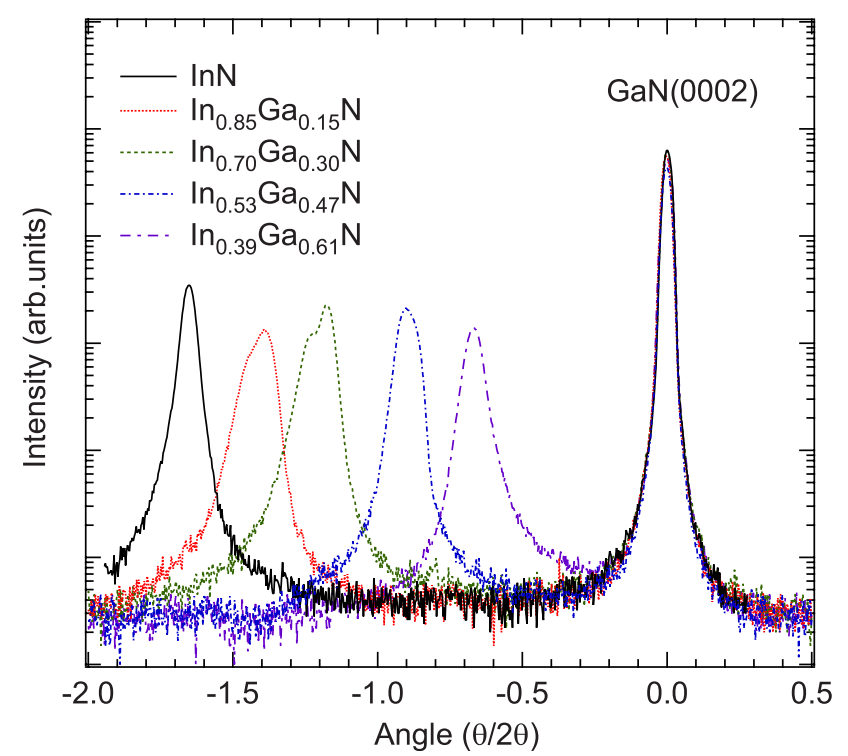

FIG. 1. (Color online) X-ray diffraction of the five $\operatorname{In}_{x} \mathrm{Ga}_{1-x} \mathrm{~N}$ samples showing the composition range to be $0.39 \leq x \leq 1.00$.

to $500 \mathrm{~nm}$ grown by MBE or metal-organic chemical vapor deposition, while samples used in this investigation are all $200 \mathrm{~nm}$ thick and were all grown by MBE.

The $\mathrm{In}_{x} \mathrm{Ga}_{1-x} \mathrm{~N}$ alloy composition was determined by $\mathrm{x}$-ray diffraction, shown in Fig. 1, using the GaN(0002) peak as a reference. Using this technique it is possible that a small overestimation of the In content occurs (less than 5\%). Nevertheless, since the samples have a high In content, they are expected to be almost fully relaxed. All of the x-ray diffraction spectra exhibit two Bragg peaks, InGaN(0002) and $\mathrm{GaN}(0002)$, which correspond to the epilayer and buffer layers, respectively. From Fig. 1 it can be seen that the In$\mathrm{GaN}(0002)$ peak for the samples defined as $\operatorname{In}_{0.85} \mathrm{Ga}_{0.15} \mathrm{~N}$ and $\mathrm{In}_{0.70} \mathrm{Ga}_{0.30} \mathrm{~N}$ is broad and appears to be composed of two peaks. This suggests that there might be a small amount of phase separation for these samples.

Oxidized samples were studied because the conventional cleaning method, ion bombardment and annealing, produces donorlike defects near the surface of InN and In-rich InGaN alloys, resulting in an increased near-surface electron concentration. ${ }^{17-20}$ Unconventional methods have been successful in cleaning both InN (Ref. 19) and GaN; ${ }^{21}$ however, cleaning InGaN alloys would require such methods to be optimized for each composition and hence cleaning is not a practical process to employ. The surface contaminants could potentially change the surface Fermi level pinning; however, previous studies suggest that the surface Fermi level shift with oxygen adsorption is negligible. $8,22-24$

XPS was utilized to determine the separation of the surface Fermi level to the valence band maximum (VBM) for the InGaN alloys. XPS measurements were performed at room temperature on a Scienta ESCA300 spectrometer at the National Centre for Electron Spectroscopy and Surface analysis, Daresbury Laboratory, UK. The samples were probed with a photon beam of energy $h \nu=1486.6 \mathrm{eV}$ produced by a monochromated rotating anode $\mathrm{Al} K \alpha$ x-ray source. The emitted photoelectrons were analyzed by a 300 $\mathrm{mm}$ radius hemispherical analyzer with a slit width of 0.8 $\mathrm{mm}$ and at a pass energy of $150 \mathrm{eV}$. The effective instrumental resolution is $0.45 \mathrm{eV}$ and is caused by a Gaussian convolution of the analyzer broadening with the natural line width of the source, $0.27 \mathrm{eV}$. The Fermi level position was calibrated using the Fermi edge of an ion bombarded silver reference sample.

Additionally In $3 d$, Ga $2 p, \mathrm{~N} 1 s, \mathrm{O} 1 s$, and $\mathrm{C} 1 s$ core levels were investigated with XPS to determine the contamination present and the native oxide layer thickness. The native oxide consisted of group III-O species but no evidence of $\mathrm{N}-\mathrm{O}$ bonding was observed. The native oxide layer thicknesses were estimated to be less than $1 \mathrm{~nm}$ on all samples from the relative intensities of the core level peaks.

Single field Hall effect measurements for these InGaN samples are influenced by parallel conduction due to the high electron concentration in the GaN:Si template and the surface space charge region. Hence Hall effect measurements would overestimate the InGaN epilayer bulk carrier concentration and thus miscalculation of the bulk Fermi level position would occur.

Consequently, to determine the position of the bulk Fermi level, the infrared reflection and optical absorption of the InGaN samples were measured using a Perkin-Elmer Spectrum GX Fourier transform infrared spectrometer and a Perkin-Elmer Lambda $25 \mathrm{UV} /$ vis spectrometer for energies below and above $1.2 \mathrm{eV}$, respectively. The reflection spectra were recorded for an incident and reflected angle of $35^{\circ}$ to the surface normal and the reflectance was determined by the ratio of reflection from the sample and from a highly reflective optical mirror.

\section{RESULTS, ANALYSIS, AND DISCUSSION}

The position of the VBM with respect to the surface Fermi level was determined by extrapolating the leading edge of the valence band photoemission spectra to the intersection with the background level to account for the finite resolution of the spectrometer, ${ }^{25}$ as shown in Fig. 2. This yields a VBM to surface Fermi level separation, $\xi$, of 1.45 $\mathrm{eV}$ for InN, which increases to $2.38 \mathrm{eV}$ for $\operatorname{In}_{0.39} \mathrm{Ga}_{0.61} \mathrm{~N}$, the least In-rich sample, as shown in Table III.

Optical absorption spectroscopy was utilized to determine the bulk Fermi level position. The infrared, visible, and ultraviolet transmissions of the InGaN samples were measured at room temperature as a function of photon energy. The transmission was converted into the absorption coefficient assuming a constant refractive index by using the Moss rule. ${ }^{26}$ Figure 3 shows the experimental absorption spectra for $\operatorname{In}_{x} \mathrm{Ga}_{1-x} \mathrm{~N}$ with $0.39 \leq x \leq 0.85$. The oscillatory structure is attributed to Fabry-Pérot interference. This Fabry-Pérot oscillation corresponds to the GaN:Si template layer thickness and continues past the absorption edge, which is consistent with these oscillations corresponding to the $3 \mu \mathrm{m}$ thick GaN:Si template layer rather than the $\operatorname{In}_{x} \mathrm{Ga}_{1-x} \mathrm{~N}$ layer.

The absorption coefficient of the InGaN samples was modeled using the one electron model considering only direct transitions between the valence band, $E_{v}$, and the conduction band, $E_{c}{ }^{27}$ and neglecting band tailing effects, 


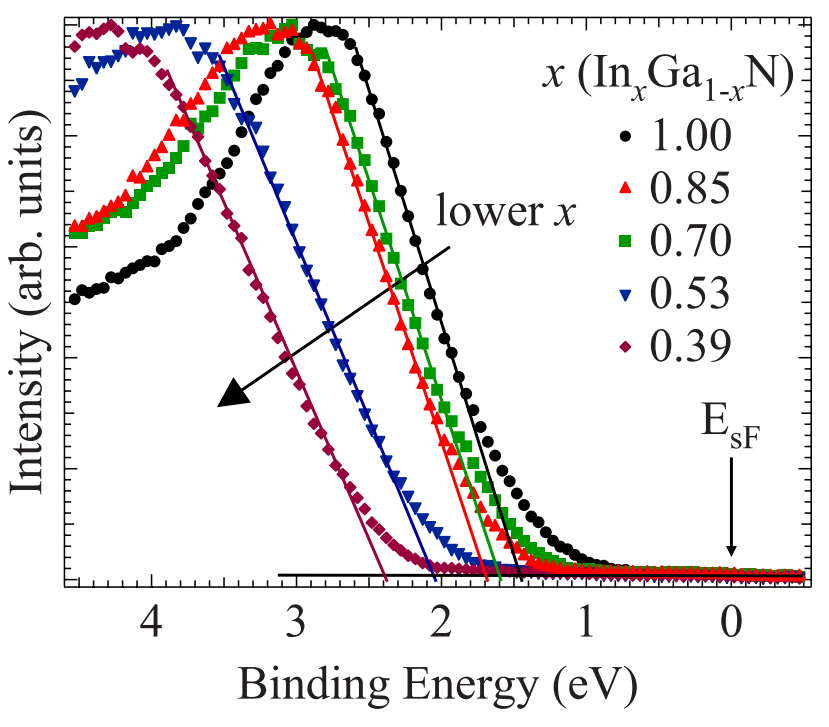

FIG. 2. (Color online) Valence band XPS spectrum for $\operatorname{In}_{x} \mathrm{Ga}_{1-x} \mathrm{~N}$ alloys with the leading edge linear fit extrapolated down to the background level. The VBM occurs at the intersection of the linear fit and the background level. The binding energy scale is with respect to the surface Fermi level, $E_{\mathrm{sF}}$. Therefore the separation between the VBM and the surface Fermi level, $\xi$, is the energy at which the intersection occurs.

$$
\alpha(\varepsilon)=\left(\frac{\pi e^{2} \hbar}{\epsilon_{0} m_{e}^{2} c \tilde{n} \varepsilon}\right) g(\varepsilon)\left[f_{\mathrm{FD}}\left(E_{v}\right)-f_{\mathrm{FD}}\left(E_{c}\right)\right],
$$

where $\epsilon_{0}$ is the permittivity of vacuum, $\tilde{n}$ is the refractive index, and $f_{\mathrm{FD}}$ is the Fermi-Dirac distribution. The density of states, $g(\varepsilon)$, is derived using the alpha approximation for the nonparabolic conduction band

$$
g(\varepsilon)=\frac{1}{2 \pi^{2}}\left(\frac{2 m_{0}^{*}}{\hbar^{2}}\right)^{3 / 2} \varepsilon^{1 / 2}\left(\frac{\varepsilon}{E_{g}}+1\right)^{1 / 2}\left(\frac{2 \varepsilon}{E_{g}}+1\right)
$$

where $E_{g}$ is the band gap and $m_{0}^{*}$ is the electron effective mass at the CBM. The InN and $\mathrm{GaN}$ band gaps at room temperature were taken to be 0.64 (Ref. 1) and $3.44 \mathrm{eV}^{2}$ respectively, and the band gap across the composition as being described by a bowing parameter $b$ of $1.7 \mathrm{eV}^{28}$ The effective masses at the CBM for InN and $\mathrm{GaN}$ were taken to

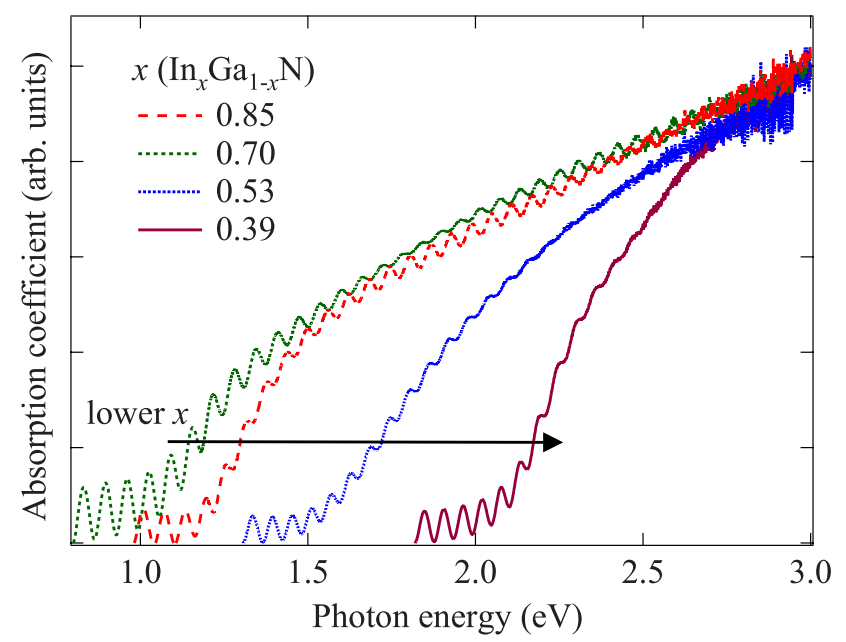

FIG. 3. (Color online) The experimental absorption spectra for $\operatorname{In}_{x} \mathrm{Ga}_{1-x} \mathrm{~N}$ with $0.39 \leq x \leq 0.85$.

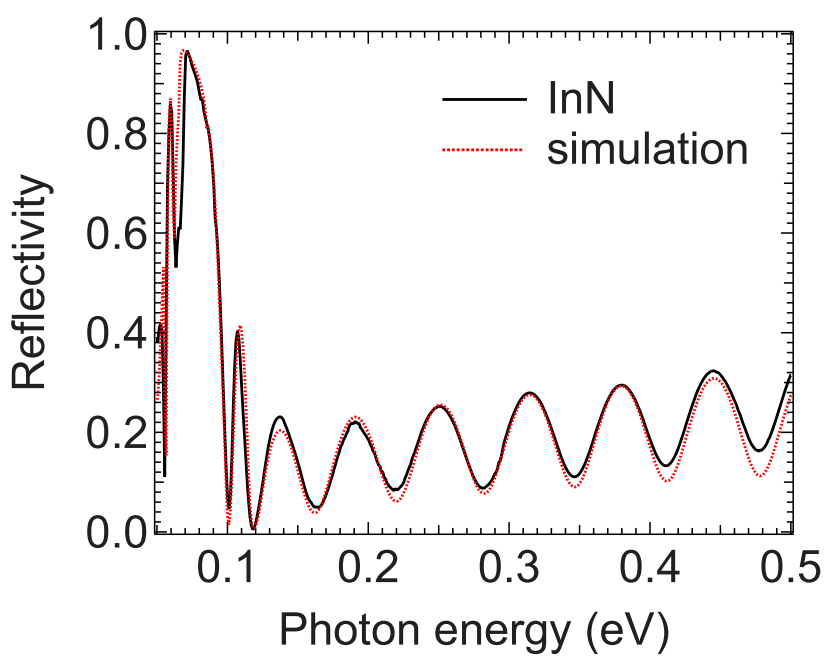

FIG. 4. (Color online) The experimental infrared reflectivity spectrum for InN plotted with the simulated spectrum.

be $0.045 m_{0}$ and $0.20 m_{0}$, respectively, and the value of $m_{0}^{*}$ as a function of composition as being described by a bowing parameter of $0.7 b=0.119 .^{29,30}$ The bulk Fermi level position can then be varied until the modeled absorption edge matches the experimental absorption edge. The bulk Fermi level values are listed in Table III.

The InN bulk Fermi level position determined by optical absorption spectroscopy was considered unreliable because of the large Urbach tailing. Therefore the optical absorption derived bulk Fermi level positions were used for the four most Ga-rich InGaN samples and infrared reflection spectroscopy was performed on InN to determine its bulk Fermi level position.

The infrared reflectivity of $\mathrm{InN}$ was measured at room temperature as a function of photon energy. Figure 4 shows the experimental and simulated infrared reflectivities for InN. The oscillations observed in the experimental spectrum are due to Fabry-Pérot interference, corresponding to the $\mathrm{GaN}: \mathrm{Si}$ template thickness.

The infrared reflectivity of a (0001) sapphire sample was also measured at various angles for modeling purposes. The dielectric function of sapphire was simulated with the factorized model ${ }^{31,32}$ to incorporate the transverse optical and longitudinal optical phonon frequencies, while the infrared reflection spectrum for the InN sample was simulated using a two-oscillator dielectric model, classical free-carrier absorption theory and a multilayer structure approximation, to incorporate the two buffer layers and the (0001) sapphire substrate. ${ }^{33,34}$ Three parameters, the phonon damping coefficient, the free-carrier lifetime, $\tau$, and the plasma frequency, $\omega_{p}$, were modified until the simulated spectral features matched those in the experimental infrared reflectance spectra. The values for the high-frequency constants, $\epsilon_{\infty}$, the static dielectric constant, $\boldsymbol{\epsilon}_{s}$, the transverse optical phonon frequency, $\omega_{\mathrm{TO}}$, and the longitudinal optical phonon frequency, $\omega_{\mathrm{LO}}$, for InN and sapphire are listed in Table I, while the values used for $\tau$ and $\omega_{p}$ for the InN layer, two buffer layers, and substrate are given in Table II.

The carrier concentration, $n$, can be calculated from the plasma frequency, $\omega_{p}$, using the relation 
TABLE I. The parameters used in the infrared reflectivity simulations, where $\epsilon_{\infty}, \epsilon_{s}, \omega_{\mathrm{TO}}$, and $\omega_{\mathrm{LO}}$ are the high-frequency and static dielectric constants and the transverse and longitudinal optical phonon frequencies, respectively.

\begin{tabular}{lcccc}
\hline \hline & & & $\omega_{\mathrm{TO}}$ & \\
& $\epsilon_{\infty}$ & $\epsilon_{s}$ & $\begin{array}{c}\omega_{\mathrm{LO}} \\
(\mathrm{meV})\end{array}$ & $(\mathrm{meV})$ \\
\hline InN $^{\mathrm{a}}$ & 6.70 & 10.50 & 59.24 & \\
Sapphire $^{\mathrm{b}}$ & 3.06 & 9.39 & 47.79 & 48.09 \\
& & & 54.53 & 59.75 \\
& & & 70.60 & 78.15 \\
& & & 78.68 & 112.47 \\
\hline \hline
\end{tabular}

${ }^{\mathrm{a}}$ References 45 and 46

${ }^{\mathrm{b}}$ Reference 32 .

$$
\omega_{p}=\left(\frac{n e^{2}}{\epsilon_{\infty} \epsilon_{0}\left\langle m^{*}\right\rangle}\right)^{1 / 2}
$$

where $\left\langle m^{*}\right\rangle$ is the density of states averaged effective mass,

$$
\left\langle m^{*}\right\rangle=\frac{m_{0}^{*}}{n} \int_{0}^{\infty}\left(1+\frac{2 E}{E_{g}}\right) g(E) f_{\mathrm{FD}}(E) d E .
$$

To calculate the bulk Fermi level position from the carrier concentration, carrier statistics are used.

To determine the location of the surface and bulk Fermi levels with respect to the branch point energy the relative position of the VBM to the branch point energy was taken to be $-1.83 \mathrm{eV}$ for InN (Ref. 15) and $-2.4 \mathrm{eV}$ for $\mathrm{GaN},{ }^{35-37}$ giving the InN/GaN valence band offset value as $0.57 \mathrm{eV}$, which is consistent with some previous studies, ${ }^{38,39}$ although there are a large variety of results reported, as discussed in detail in Ref. 39. The valence band offset is expected to vary linearly with the composition. ${ }^{37}$

Figure 5 shows the VBM, CBM, and bulk and surface Fermi level positions across the composition range with respect to the branch point energy. Both the surface and bulk Fermi level positions are below the branch point energy for the In-rich InGaN alloys. This allows the existence of unoccupied donorlike surface states that have donated their electrons into the surface electron accumulation layer. This is also consistent with the downward band bending ranging from 0.19 to $0.66 \mathrm{eV}$. For the $\mathrm{In}_{0.39} \mathrm{Ga}_{0.61} \mathrm{~N}$ alloy, the surface Fermi level is located $0.2 \mathrm{eV}$ above the branch point energy, indicating a surface electron depletion layer is present but the downward band bending of $0.19 \mathrm{eV}$ indicates that this sample exhibits a surface electron accumulation layer. Any uncertainty in the surface and bulk Fermi level positions will result in a wider range of possible surface sheet charge den-

TABLE II. The variable parameters used in the infrared reflectivity simulations, where $\tau$ and $\omega_{p}$ are the free-carrier lifetime and the plasma frequency, respectively.

\begin{tabular}{lcc}
\hline \hline & $\begin{array}{c}\tau \\
(\mathrm{ps})\end{array}$ & $\omega_{p}(\mathrm{meV}) \pm 25 \mathrm{meV}$ \\
\hline $\mathrm{InN}$ & 0.83 & 112 \\
$\mathrm{GaN}$ & 3.33 & 1 \\
$\mathrm{GaN}: \mathrm{Si}$ & 0.20 & 62 \\
Sapphire & 0.67 & 0 \\
\hline \hline
\end{tabular}

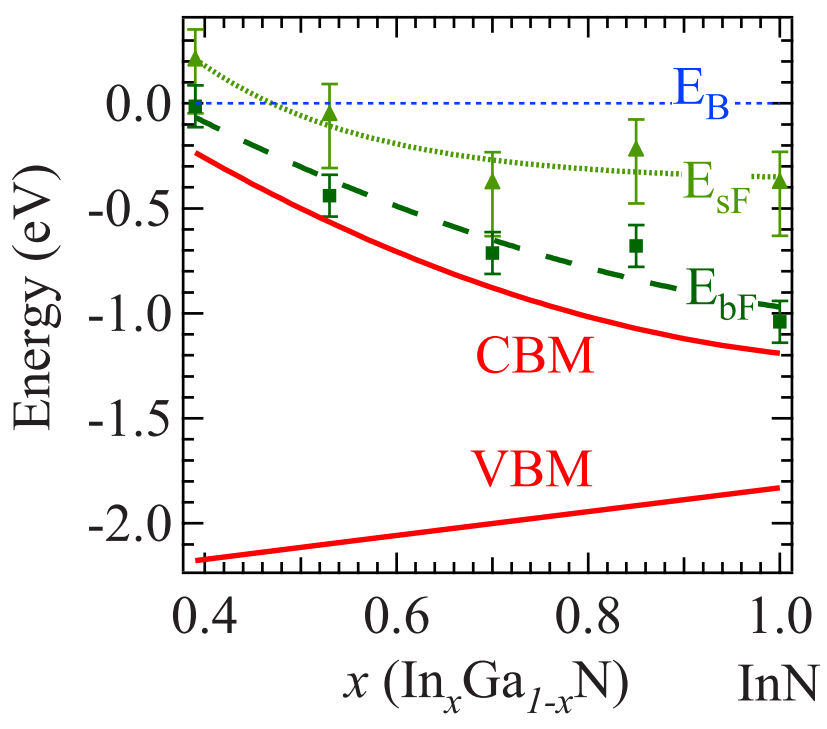

FIG. 5. (Color online) The CBM and the VBM of $\operatorname{In}_{x} \mathrm{Ga}_{1-x} \mathrm{~N}$ as a function of $x$ with respect to the branch point energy, $E_{B}$. The band gap bowing is shown entirely in the CBM as the separation of the VBM and the branch point energy is expected to vary linearly with composition. The relative positions of the surface and bulk Fermi levels, $E_{\mathrm{sF}}$ and $E_{\mathrm{bF}}$, that were determined by XPS and infrared reflection and optical absorption spectroscopies, as a function of $x$, are also shown as triangular and square points, respectively. The dashed (dotted) line is a guide to the eyes for the bulk (surface) Fermi level position.

sities and within the error bars the surface Fermi level could be at the branch point energy and this sample could exhibit no band bending.

From Fig. 5 it also appears that the surface Fermi level is pinned closer to the branch point energy with increasing $\mathrm{Ga}$ fraction. For a simplistic approximation the change from an accumulation layer to a depletion layer would be marked with both the surface and bulk Fermi levels intersecting the branch point energy at the same composition. However, the branch point energy is defined as the crossover point from states that are predominantly acceptorlike to predominantly donorlike. Therefore it is possible for some donorlike surface states to exist just above the branch point energy. Hence downward band bending can still occur when the surface and bulk Fermi levels are slightly above the branch point energy.

Hence the exact character of the $\mathrm{In}_{0.39} \mathrm{Ga}_{0.61} \mathrm{~N}$ sample surface charge region will be dependent on the exact location of the surface and bulk Fermi levels. All of these observations above indicate that, for similar bulk Fermi levels, the transition from an accumulation layer at the surface of Inrich $\operatorname{In}_{x} \mathrm{Ga}_{1-x} \mathrm{~N}$ to a depletion layer at the surface of Ga-rich $\mathrm{In}_{x} \mathrm{Ga}_{1-x} \mathrm{~N}$ will be at a composition of slightly less than $x$ $=0.39$. This result, obtained from the relative positions of the bulk and surface Fermi levels, occurs at a slightly less In-rich composition than the intersection of the surface Fermi level best fit line with the branch point energy, which is a commonly used method of approximating the composition at which the transition occurs, as seen in previous studies. ${ }^{8,12}$ Nonetheless, the surface charge layer character for an individual InGaN sample will be dependent on its particular surface and bulk Fermi level locations.

The band bending and carrier concentration profiles as a function of depth from the surface were evaluated by solving 

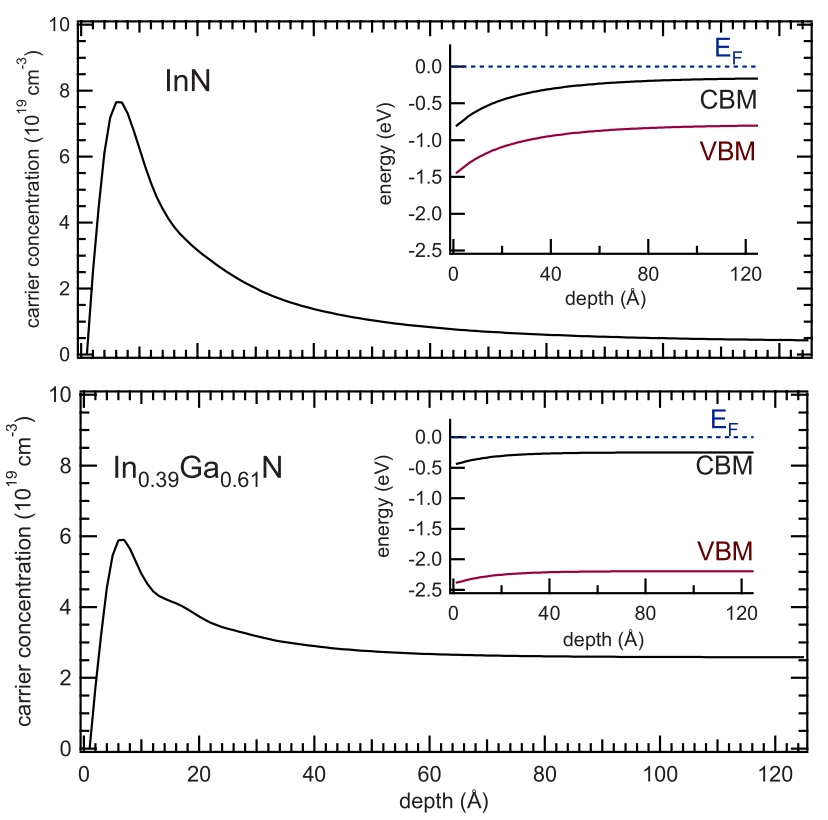

FIG. 6. (Color online) The carrier concentration as a function of depth from the semiconductor surface for $\operatorname{InN}$ and $\operatorname{In}_{0.39} \mathrm{Ga}_{0.61} \mathrm{~N}$. Inset: the CBM and VBM positions with respect to the Fermi level, $E_{F}$, as a function of depth from the semiconductor surface.

Poisson's equation ${ }^{40}$ within a MTFA. ${ }^{9,24,41-44}$ The highfrequency dielectric constant for $\mathrm{GaN}$ was taken to be $5.35,{ }^{32}$ and it was assumed that $\epsilon_{\infty}$ varies linearly with composition. ${ }^{45}$ Figure 6 shows the band bending and carrier concentration profile for $\mathrm{InN}$ and $\mathrm{In}_{0.39} \mathrm{Ga}_{0.61} \mathrm{~N}$. From Fig. 6, it can be seen that the extent of downward band bending and near-surface carrier concentration is higher with a larger In content. Also, from Poisson's equation, the surface sheet charge density can be calculated from the gradient of the band bending potential at the surface. ${ }^{43}$ The surface sheet charge density varies from $5.0 \times 10^{12}$ to $1.5 \times 10^{13} \mathrm{~cm}^{-2}$, see Table III, confirming the presence of a surface electron accumulation layer in the $\operatorname{In}_{x} \mathrm{Ga}_{1-x} \mathrm{~N}$ samples with a composition range of $0.39 \leq x \leq 1.00$ and that this electron accumulation layer is more extreme in the most In-rich InGaN samples.

The XPS determined surface Fermi level position may be slightly underestimated when there is significant downward band bending since the measured surface Fermi level is averaged over a finite depth; $95 \%$ of the signal originates from approximately $72 \AA$ as the inelastic mean free path of electrons with a kinetic energy of $1485 \mathrm{eV}$ is $\sim 24 \AA$ in
$\mathrm{In}_{x} \mathrm{Ga}_{1-x} \mathrm{~N}$. XPS is surface sensitive because the photoelectrons undergo inelastic scattering in the bulk of the sample. The probability of a photoelectron being detected without energy loss has an exponential depth dependence, which means that the photoelectrons from the surface dominate the signal. From Fig. 6 it can be seen that the CBM and VBM rapidly bend near the surface of the semiconductor over a distance shorter than the XPS sampling range. Therefore the Fermi level rapidly changes as a function of depth from the surface. This small underestimation means that the surface electron accumulation could be slightly more extreme than predicted here by Poisson's equation within a MTFA using XPS determined surface Fermi level positions.

The trend observed in this study, of the band bending to become less extreme and the size of the surface sheet charge density to decrease with increasing Ga content in the InGaN samples, is consistent with previous reports into the transition from surface electron accumulation in $\mathrm{InN}$ to depletion in $\mathrm{GaN}^{8}$ However, the transition from a surface electron accumulation layer to a surface electron depletion layer is found to be at a lower In composition than previously determined. Also the values for CBM, VBM, band gap energy, and valence band offset produce carrier concentration and surface sheet charge density values that are in agreement with earlier literature for $\mathrm{InN},{ }^{8,10,12}$ although, in these earlier studies, the bulk Fermi level was either not determined or overestimated on the basis of single field Hall effect measurements.

The presence of a surface electron accumulation layer in In-rich InGaN will have a profound effect on electronic properties. For example, only Ohmic contacts will be formed at metal/In-rich InGaN interfaces and hence this will impact on the potential device types. The inherent nature of the surface accumulation layer in In-rich InGaN, resulting from the surface and bulk Fermi level positions, will make controlling or eliminating the space charge layer easier for more Ga-rich InGaN alloys. Therefore the most effective applications for In-rich InGaN may well be those that exploit the surface electron accumulation layer.

\section{CONCLUSIONS}

The positions of the surface and bulk Fermi levels for $\mathrm{In}_{x} \mathrm{Ga}_{1-x} \mathrm{~N}$ with a composition range of $0.39 \leq x \leq 1.00$ have been measured by XPS and infrared reflection and optical absorption spectroscopies and compared to the position of the branch point energy. The differences in the surface and

TABLE III. The values of the surface and bulk Fermi levels with respect to the VBM, $E_{\mathrm{sF}}$ and $E_{\mathrm{bF}}$, respectively, band bending, $V_{\mathrm{bb}}$, bulk carrier concentration, bulk $n$, and surface sheet charge density, $n_{\mathrm{ss}}$, that have been determined for the $\mathrm{In}_{x} \mathrm{Ga}_{1-x} \mathrm{~N}$ samples.

\begin{tabular}{lccccc}
\hline \hline Sample & $\begin{array}{c}E_{\mathrm{sF}} \\
(\mathrm{eV})\end{array}$ & $\begin{array}{c}E_{\mathrm{bF}} \\
(\mathrm{eV})\end{array}$ & $\begin{array}{c}V_{\mathrm{bb}} \\
(\mathrm{eV})\end{array}$ & $\begin{array}{c}\text { bulk } n \\
\left(10^{18} \mathrm{~cm}^{-3}\right)\end{array}$ & $\begin{array}{c}n_{\mathrm{ss}} \\
\left(10^{12} \mathrm{~cm}^{-2}\right)\end{array}$ \\
\hline $\mathrm{InN}$ & 1.45 & 0.79 & 0.66 & 3.73 & 15.10 \\
$\mathrm{In}_{0.85} \mathrm{Ga}_{0.15} \mathrm{~N}$ & 1.69 & 1.24 & 0.45 & 25.54 & 13.66 \\
$\mathrm{In}_{0.70} \mathrm{Ga}_{0.30} \mathrm{~N}$ & 1.62 & 1.30 & 0.32 & 7.61 & 6.55 \\
$\mathrm{In}_{0.53} \mathrm{Ga}_{0.47} \mathrm{~N}$ & 2.04 & 1.68 & 0.36 & 8.38 & 8.17 \\
$\mathrm{In}_{0.39} \mathrm{Ga}_{0.61} \mathrm{~N}$ & 2.38 & 2.19 & 0.19 & 25.82 & 4.96 \\
\hline \hline
\end{tabular}


bulk Fermi level positions were used to calculate the extent of the band bending and the surface sheet charge density by solving Poisson's equation within a MTFA. The most In-rich InGaN samples exhibit extreme downward band bending and a high surface sheet charge density, whereas for the least In-rich sample, $\mathrm{In}_{0.39} \mathrm{Ga}_{0.61} \mathrm{~N}$, the band bending is much less and the surface sheet charge density is lower.

\section{ACKNOWLEDGMENTS}

We are grateful to Danny Law and Graham Beamson of NCESS for their assistance with the XPS measurements. Also, we acknowledge the Engineering and Physical Sciences Research Council, U.K. for financial support under Grant No. EP/E031595/1. ISOM would like to acknowledge financial support from the regional government of Comunidad de Madrid, Project No. S-0505/AMB/0374 (20052009). Warwick and ISOM also acknowledge support from RAINBOW, a Marie Curie Initial Training Network funded by the European Commission under the 7th Framework program.

${ }^{1}$ J. Wu, W. Walukiewicz, W. Shan, K. M. Yu, J. W. Ager III, S. X. Li, E. E. Haller, H. Lu, and W. J. Schaff, J. Appl. Phys. 94, 4457 (2003).

${ }^{2}$ I. Vurgaftman and J. R. Meyer, J. Appl. Phys. 94, 3675 (2003).

${ }^{3}$ J. Wu, W. Walukiewicz, K. M. Yu, J. W. Ager III, E. E. Haller, H. Lu, and W. J. Schaff, Appl. Phys. Lett. 80, 4741 (2002).

${ }^{4}$ W. Walukiewicz, J. W. Ager III, K. M. Yu, Z. Liliental-Weber, J. Wu, S. X. Li, R. E. Jones, and J. D. Denlinger, J. Phys. D 39, R83 (2006).

${ }^{5}$ F. A. Ponce and D. P. Bour, Nature (London) 386, 351 (1997).

${ }^{6}$ V. M. Polyakov and F. Schwierz, Appl. Phys. Lett. 88, 032101 (2006).

${ }^{7}$ Z. Liliental-Weber, R. E. Jones, H. C. M. van Genuchten, K. M. Yu, W. Walukiewicz, J. W. Ager III, E. E. Haller, H. Lu, and W. J. Schaff, Physica B 401-402, 646 (2007).

${ }^{8}$ T. D. Veal, P. H. Jefferson, L. F. J. Piper, C. F. McConville, T. B. Joyce, P. R. Chalker, L. Considine, H. Lu, and W. J. Schaff, Appl. Phys. Lett. 89, 202110 (2006).

${ }^{9}$ P. D. C. King, T. D. Veal, P. H. Jefferson, C. F. McConville, H. Lu, and W. J. Schaff, Phys. Rev. B 75, 115312 (2007).

${ }^{10}$ I. Mahboob, T. D. Veal, L. F. J. Piper, C. F. McConville, H. Lu, W. J. Schaff, J. Furthmüller, and F. Bechstedt, Phys. Rev. B 69, 201307(R) (2004).

${ }^{11}$ I. Mahboob, T. D. Veal, C. F. McConville, H. Lu, and W. J. Schaff, Phys. Rev. Lett. 92, 036804 (2004).

${ }^{12}$ P. D. C. King, T. D. Veal, H. Lu, P. H. Jefferson, S. A. Hatfield, W. J. Schaff, and C. F. McConville, Phys. Status Solidi B 245, 881 (2008).

${ }^{13}$ H. Lu, W. J. Schaff, L. F. Eastman, and C. E. Stutz, Appl. Phys. Lett. 82, 1736 (2003)

${ }^{14}$ M. Noguchi, K. Hirakawa, and T. Ikoma, Phys. Rev. Lett. 66, 2243 (1991).

${ }^{15}$ P. D. C. King, T. D. Veal, P. H. Jefferson, S. A. Hatfield, L. F. J. Piper, C. F. McConville, F. Fuchs, J. Furthmüller, F. Bechstedt, H. Lu, and W. J.
Schaff, Phys. Rev. B 77, 045316 (2008).

${ }^{16}$ L. F. J. Piper, T. D. Veal, M. J. Lowe, and C. F. McConville, Phys. Rev. B 73, 195321 (2006).

${ }^{17}$ T. Ohashi, Y. Saito, T. Maruyama, and Y. Nanishi, J. Cryst. Growth 237239, 1022 (2002).

${ }^{18}$ L. F. J. Piper, T. D. Veal, C. F. McConville, H. Lu, and W. J. Schaff, Phys. Status Solidi C 3, 1841 (2006).

${ }^{19}$ L. F. J. Piper, T. D. Veal, M. Walker, I. Mahboob, C. F. McConville, H. Lu, and W. J. Schaff, J. Vac. Sci. Technol. A 23, 617 (2005).

${ }^{20}$ L. F. J. Piper, T. D. Veal, C. F. McConville, H. Lu, and W. J. Schaff, Appl. Phys. Lett. 88, 252109 (2006).

${ }^{21}$ V. M. Bermudez, T. M. Jung, K. Doverspike, and A. E. Wickenden, J. Appl. Phys. 79, 110 (1996).

${ }^{22}$ V. M. Bermudez, J. Appl. Phys. 80, 1190 (1996).

${ }^{23}$ R. A. Beach, E. C. Piquette, and T. C. McGill, MRS Internet J. Nitride Semicond. Res. 4S1, G6.26 (1999).

${ }^{24}$ P. D. C. King, T. D. Veal, C. F. McConville, F. Fuchs, J. Furthmüller, F. Bechstedt, P. Schley, R. Goldhahn, J. Schörmann, D. J. As, K. Lischka, D. Muto, H. Naoi, Y. Nanishi, H. Lu, and W. J. Schaff, Appl. Phys. Lett. 91, 092101 (2007).

${ }^{25}$ S. A. Chambers, T. Droubay, T. C. Kaspar, and M. Gutowski, J. Vac. Sci. Technol. B 22, 2205 (2004).

${ }^{26}$ J. I. Pankove, Optical Processes in Semiconductors (Dover, New York, 1975).

${ }^{27}$ H. C. Casey, Jr. and F. Stern, J. Appl. Phys. 47, 631 (1976).

${ }^{28}$ P. Schley, R. Goldhahn, A. T. Winzer, G. Gobsch, V. Cimalla, O. Ambacher, H. Lu, W. J. Schaff, M. Kurouchi, Y. Nanishi, M. Rakel, C. Cobet, and N. Esser, Phys. Rev. B 75, 205204 (2007).

${ }^{29}$ B. R. Nag, Phys. Status Solidi B 237, R1 (2003).

${ }^{30}$ L. F. J. Piper, T. D. Veal, I. Mahboob, C. F. McConville, H. Lu, and W. J. Schaff, Phys. Rev. B 70, 115333 (2004).

${ }^{31}$ M. Schubert, T. E. Tiwald, and C. M. Herzinger, Phys. Rev. B 61, 8187 (2000).

${ }^{32}$ G. Yu, N. L. Rowell, and D. J. Lockwood, J. Vac. Sci. Technol. A 22, 1110 (2004).

${ }^{33}$ C. Pickering, J. Phys. C 13, 2959 (1980).

${ }^{34}$ T. Abe and T. Kato, Jpn. J. Appl. Phys. 4, 742 (1965).

${ }^{35}$ H. Lüth, Phys. Status Solidi A 187, 33 (2001).

${ }^{36}$ T. U. Kampen and W. Mönch, Appl. Surf. Sci. 117-118, 388 (1997).

${ }^{37}$ W. Mönch, J. Appl. Phys. 80, 5076 (1996).

${ }^{38}$ S. H. Wei and A. Zunger, Appl. Phys. Lett. 69, 2719 (1996).

${ }^{39}$ P. D. C. King, T. D. Veal, C. E. Kendrick, L. R. Bailey, S. M. Durbin, and C. F. McConville, Phys. Rev. B 78, 033308 (2008).

${ }^{40} \mathrm{~W}$. Mönch, Semiconductor Surfaces and Interfaces, 3rd ed. (Springer, Berlin, 2001).

${ }^{41}$ H. Übensee, G. Paasch, and J.-P. Zöllner, Phys. Rev. B 39, 1955 (1989).

${ }^{42}$ J. P. Zöllner, H. Übensee, G. Paasch, T. Fiedler, and G. Gobsch, Phys. Status Solidi B 134, 837 (1986).

${ }^{43}$ T. D. Veal, L. F. J. Piper, W. J. Schaff, and C. F. McConville, J. Cryst. Growth 288, 268 (2006).

${ }^{44}$ T. D. Veal, L. F. J. Piper, M. R. Phillips, M. H. Zareie, H. Lu, W. J. Schaff, and C. F. McConville, Phys. Status Solidi A 204, 536 (2007).

${ }^{45}$ T. Inushima, M. Higashiwaki, and T. Matsui, Phys. Rev. B 68, 235204 (2003).

${ }^{46}$ W. Walukiewicz, R. E. Jones, S. X. Li, K. M. Yu, J. W. Ager III, E. E. Haller, H. Lu, and W. J. Schaff, J. Cryst. Growth 288, 278 (2006). 